Abstracts*

\title{
$51^{\text {th }}$ National Congress of the Italian Society of Clinical Biochemistry and Clinical Molecular Biology (SIBioC - Laboratory Medicine)
}

\author{
Padova Fiere, Padua (Italy) \\ 20-22 November 2019
President of the Congress
Mario Plebani (Padova) \\ Scientific Committee \\ Giuseppe Lippi (Verona) \\ Sabrina Buoro (Bergamo)
}

Fiamma Balboni, Umberto Basile, Sergio Bernardini, Sabrina Buoro, Marcello Ciaccio, Aldo Clerico,Antonio D'Avolio, Giorgio Da Rin, Davide Farci Santarcangeli, Maria Stella Graziani,

Giuseppe Lippi, Silvia Mengozzi, Giampaolo Merlini, Martina Montagnana, Andrea Mosca,

Michele Mussap, Antonello Nonnato, Roberta Pacifici, Silvia Pichini, Mario Plebani,

Giulia Sancesario, Laura Sciacovelli, Tommaso Trenti, Martina Zaninotto

\section{Organising Committee}

Giorgio Da Rin, Michele Mussap, Mario Plebani, Laura Sciacovelli, Martina Zaninotto

\section{Scientific Secretariat}

Sabrina Buoro, Giorgio Da Rin, Laura Sciacovelli, Giuseppe Lippi, Michele Mussap, Mario Plebani, Martina Zaninotto

\footnotetext{
*) These abstracts have been reproduced directly from the material supplied by the authors, without editorial alteration by the staff of this Journal. Insufficiencies of preparation, grammar, spelling, style, syntax, and usage are the authors.
} 


\title{
LABORATORY MEDICINE APPLIED TO THE USE OF MEDICAL AND LIGHT CANNABIS
}

\author{
F. P. Busardò̀ ${ }^{1}$ S. Pichini ${ }^{2}$ \\ ${ }^{1}$ Department of Excellence-Biomedical Sciences and Public Health, Ancona, Italy \\ ${ }^{2}$ National Centre on Addiction and Doping Istituto Superiore di Sanità, Rome, Italy
}

From an analytical point of view, the difference between medical cannabis and cannabis light is in the percentage of the pharmacologically active cannabinoids. The Italian FM 2 medical product contains about $6 \%$ $\Delta^{9}$-tetrahydrocannabinol (THC) and $8 \%$ cannabidiol (CBD). Conversely, in the light cannabis THC is generally less than $0.2 \%$ or in some specific cases less than $0.4 \%$ while CBD can range from $2 \%$ to $40 \%$. The use of these two products is completely different. Medical cannabis is a symptomatic treatment mainly used for neuropathic pain in multiple sclerosis as well as peripheral neuropathic pain, rheumatoid arthritis, sleep disturbances and depression and intractable cancer pain. Light cannabis is currently a free sale product, used principally for the properties of CBD, since THC is quite absent. Indeed, CBD is not psychotropic but it is psychoactive because it is a major miorelaxant and humour tranquillizer relaxant with no abuse liability. Nevertheless, it causes drowsiness, a symptom that should dangerous in normal daily activities such as driving or working.

Screening tests on biological fluids from consumers of medical cannabis clearly give a positive result, which goes to confirmatory chromatographic analysis. This latter provides cannabinoids concentrations which cannot be distinguished by those obtained after consuming recreational cannabis. In medico-legal situations (eg. workplace drug testing, driving under the influence of drug, etc.) only medical prescription can exonerate an individual from the charge of illegal cannabis use.

Conversely, due to the very low THC concentration achieved after consuming light cannabis, typical screening tests can result either negative (eg. after a single product consumption) or positive (eg. after repeated consumption) as a function of test cut-off. In case of positivity to these test, the confirmatory chromatographic analysis will disclose very low THC concentration in any of eventual analysed biological fluid (oral fluid, blood or urine) and higher concentrations of CBD.

For this reason, THC/CBD concentration ratios in serum, blood and oral fluid or THC-COOH/CBD concentration ratio in urine never exceeding the mean value of 0.9 might be a useful biomarker to identify use of light cannabis vs that of illegal THC cannabis, where the THC/CBD concentration ratios are generally greater than 10 or vs that of medical cannabis where ratios are greater than 2 .

\section{DIAGNOSTIC ALGORITHMS ON THE WEB: HOW RELIABLE ARE THEY?}

\section{Giavarina}

Clinical Laboratory, St. Bortolo Hospital, AULSS n.8 Berica, Vicenza

The concept of algorithm has existed for centuries. An algorithm is a step-by-step method of solving a problem. Diagnosing is a problem to solve, and it is helpful to have unambiguous specifications for performing an automated reasoning to achieve the solution: the diagnosis. For decades, algorithms have been proposed in many fields of diagnostic and therapeutic medicine. In Laboratory Medicine their use are particularly frequent, given the availability of quantitative data, reference limits and cut-off. Only for instance, the Mayo Clinic publish more than 120 different diagnostic algorithms, in its website (1). Many websites offer reliable algorithms, most of them under the endorsement of scientific societies.

Nevertheless, Web is a democratic area, where everyone can write, load and propose everything. However, science is not democratic, nor is medicine, laboratory too. Therefore, the first problem is the same for the scientific information: how recognizing solid and reliable diagnostic algorithms on the Web, and which algorithms to choose between many options. The solutions are the usual ones: Evidence Base Medicine, involving experts, evidences and patients.

A second problem is the new social nature of the Web, the evolution of the interactions between Web and users. We are now in a transition era, the 2.0 Web, moving towards the Web 3.0, the so-called semantic Web. The most significant difference between Web 2.0 and 3.0 in the World Wide Web is greater collaboration 
among Internet users, content providers and enterprises. Users have now more input into the nature and scope of Web content and in some cases exert real-time control over it. The problem is that, to obtain a good output from algorithms, or from an artificial intelligence ( $\mathrm{Al}$ ), a good input needs. Algorithms used by experts could perform better that the same ones used by patients. An expert mediation between patient and web may be the winning move, as shown by the information sites, like Lab Tests Online (LTO), Mayo Clinic Laboratories, etc. The third problem is the incoming of Al. Web 4.0 coming, in which intelligent agents and smart things will be connected with the social environment. A learning machine, reading data from the same patients, big data and any available network data, could develop new diagnostic algorithms (2,3). Probably human intelligence will be not able to read or understand the "steps" of those algorithms; it can only look at the input and the output. All this can be a little distressing. Nevertheless, the main question is "are or will be these algorithms reliable for medical application and diagnosis"?

The preliminary data seem to confirm (4) this statement. Furthermore, a huge amount of data and information actually available require information technologies to be analyzed, processed and evaluated quickly. Not just Information Technology (IT), but Al will be needed for more and more personalized medicine.

Challenging the efficacy of this or that automatic algorithm, or holding that human thought can still bring to synthesis a constantly growing amount of data and knowledge is at least unrealistic, if not fool. Like Web 4.0, we are moving towards a Medicine 4.0 (5). This is probably a potential risk to the role of "human" in medicine, as it will be in all other intellect activities of our species. Nevertheless, it is also an incredible opportunity to think beyond the limits of our brain and to demonstrate the superiority of humans over machines.

\section{REFERENCES}

1. Testing Algorithms. Mayo Clinic Laboratories. https://www.mayocliniclabs.com/articles/resources/algorithms.html (last visit July 2019).

2. Gunčar G, Kukar M, Notar M, Brvar M, Černelč $P$, Notar M, Notar M. An application of machine learning to haematological diagnosis. Sci Rep 2018;11:411.

3. Rajkomar A, Oren E, Chen K, et al. Scalable and accurate deep learning with electronic health records. NPJ Digit Med 2018;1:18.

4. Piazzolla A. Gli algoritmi sono meglio dei dottori? https://forbes.it/2018/05/25/gli-algoritmi-sono-meglio-dei-dottori/ (last visit July 2019).

5. Wolf B, Scholze C. "Medicine 4.0"-The role of electronics, information technology and microsystems in modern medicine https://www.degruyter.com/view/j/cdbme.2017.3.issue-2/cdbme-2017-0038/cdbme-2017-0038.xml (last visit July 2019).

\section{LABORATORY MEDICINE AND FRAILTY}

\section{G. Lippi}

Section of Clinical Biochemistry, University of Verona, Verona, Italy

The FRAILOMIC consortium, an international coalition established for identifying predictors of frailty and disability, has endorsed a concrete definition of frailty, as "age-associated syndrome, characterized by decreased biological reserve and resistance to stressors due to functional decline of several physiological systems, which places the individual at enhanced risk of disability, hospitalization and death" (1). This straightforward definition includes essential psychological, social and cognitive factors, along with additional issues related to lifestyle factors, comorbidities and interplays among all variables.

It is now clear that frailty can be efficiently prevented or delayed by establishing appropriate interventions. Like many other human diseases, laboratory medicine can play a substantial role in this clinical setting, by providing an armamentarium of genetic, epigenetic and phenotypic tests which would help identifying subjects at higher risk of becoming frail, even at the pre-frail stage (2). Many important investigations have contributed to dissect predictive, diagnostic and even prognostic biomarkers of frailty, such as vitamin D, cortisol, testosterone, troponin, creatinine, cytokines and other inflammatory mediators. The FRAILOMIC consortium (available at: http://www.frailomic.org/) has then been created and funded under the European FP7 framework for providing further insights into the relationship between laboratory medicine and frailty (3). Briefly, FRAILOMIC 
encompasses many universities and clinical or research laboratories, engaged to create an European network which will define clinical instruments and validate measurable biomarkers for prevention, diagnosis and management of frailty. A paramount number of genetic, epigenetic and phenotypic biomarkers have been measures in several thousands of individuals. The clinical performance of these biomarkers has then been evaluated against analytical specifications and clinical outcomes. A selected set of these biomarkers has then been validated prospectively, by means of a best fit model, which enables to drive the further definition of discrete test panels that could be employed in clinical practice, with expectations to lower the clinical, social and economic impact of frailty.

\section{REFERENCES}

1. Erusalimsky JD, Grillari J, Grune T, Jansen-Duerr P, Lippi G, Sinclair AJ, et al. In Search of 'Omics'-Based Biomarkers to Predict Risk of Frailty and Its Consequences in Older Individuals: The FRAILOMIC Initiative. Gerontology 2016;62:182-90.

2. Danese E, Montagnana M, Lippi G. Proteomics and frailty: a clinical overview. Expert Rev Proteomics 2018;15:657-64.

3. Lippi G, Jansen-Duerr P, Viña J, Durrance-Bagale A, Abugessaisa I, Gomez-Cabrero D, et al. Laboratory biomarkers and frailty: presentation of the FRAILOMIC initiative. Clin Chem Lab Med 2015;53:e253-5.

\section{THE ROLE OF CLINICAL LABORATORY IN THE ASSESSMENT OF FRAILTY IN MULTIPLE MYELOMA AND AL AMYLOIDOSIS}

\section{P. Milani}

Amyloidosis Research and Treatment Center, Foundation IRCCS Policlinico San Matteo, Department of Molecular Medicine, University of Pavia, Pavia, Italy.

Multiple myeloma (MM) and light chain (AL) amyloidosis manifest with organ damage. This is caused by the tumor bulk and the organ toxicity of the monoclonal component in multiple myeloma and AL amyloidosis respectively. Organ damage makes patients particularly susceptible to the toxicity of chemotherapy. Thus, very effective agents are changing the therapeutic armamentarium for these diseases, we still need simple, objective tools to assess frailty in those subjects towards tailored, and risk adapted therapy.

In multiple myeloma, the biologic and genetic prognostic factors are useful predictors of prognosis and are combined in the International Staging System, but are still insufficient to clearly identify "frail subjects". In this context, the International Myeloma Working Group (IMWG) defined a "frailty score" based on the combination of age, functional status and comorbidities that was able to predict survival and toxicity, and determine the tolerability of therapy (1). Subsequently, the Revised Myeloma Comorbidity Index (R-MCl) provided an alternative tool for assessing frailty considering renal and lung functions, age, Karnofsky index and cytogenetics data (2). Those scores are characterized by the lack of objectives measures, they are time consuming and it could be a challenge in a busy clinical practice to incorporate all of the frailty assessments proposed. Thus, we proposed a frailty score based on the $\mathrm{N}$-terminal fragment of natriuretic peptide type $\mathrm{B}$ (NT-proBNP) in combination with performance status (ECOG) and age (3). The use of the biomarker based approach was also showed in different subsequent studies in newly diagnosed multiple myeloma patients.

In $\mathrm{AL}$ amyloidosis the possible contribution of geriatric assessment scores has not yet been validated. However, the choice of anti-plasma cell therapy is based on the evaluation of patient's fitness, mostly based on cardiac biomarkers staging. Serum levels of NT-proBNP and cardiac troponins form the basis for a staging system introduced in 2004 by the Mayo group (4) that allowed the identification of three groups with a significant difference in survival outcome and it is used for risk stratification in clinical trials. Subsequently, an European collaboration defined "extremely-fragile" patients (NT-proBNP >8500 ng/L and systolic blood pressure $<100 \mathrm{mmHg}$ at diagnosis) who still represent an unmet need for treating physicians (5).

In conclusion, in both multiple myeloma and AL amyloidosis a biomarker-based approach for the identification of frail subjects has been proposed. Further studies are needed in order to create a uniform definition for frailty in multiple myeloma and identify the most effective combination of the actual proposed scores. 


\title{
REFERENCES
}

1. Palumbo A, Bringhen S, Mateos MV, Larocca A, Facon T, Kumar SK, et al. Geriatric assessment predicts survival and toxicities in elderly myeloma patients: an International Myeloma Working Group report. Blood. 2015;125(13):2068-74.

2. Engelhardt M, Domm AS, Dold SM, Ihorst G, Reinhardt H, Zober A, et al. A concise revised Myeloma Comorbidity Index as a valid prognostic instrument in a large cohort of 801 multiple myeloma patients. Haematologica. 2017; 102(5):910-21.

3. Milani P, Vincent Rajkumar S, Merlini G, Kumar S, Gertz MA, Palladini G, et al. N-terminal fragment of the type$B$ natriuretic peptide (NT-proBNP) contributes to a simple new frailty score in patients with newly diagnosed multiple myeloma. Am J Hematol. 2016;91(11):1129-34.

4. Dispenzieri A, Gertz MA, Kyle RA, Lacy MQ, Burritt MF, Therneau TM, et al. Serum cardiac troponins and Nterminal pro-brain natriuretic peptide: a staging system for primary systemic amyloidosis. J Clin Oncol. 2004;22(18):3751-7.

5. Wechalekar AD, Schonland, S. O., Kastritis, E. et al. European Collaborative Study of treatment outcomes in 347 patients with systemic AL amyloidosis with Mayo Stage III disease. Blood. 2011;Abstract 995-ASH Meeting 2011.

\section{ESId (EARLY SEPSIS INDICATOR): EVALUATION OF THE IMPLEMENTATION IN THE "SEPSIS PATH" DEVELOPED BY REGIONAL HEALTH AGENCY OF TUSCANY}

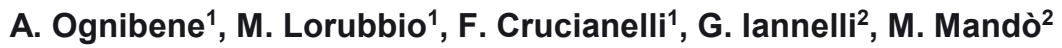 \\ ${ }^{1}$ Laboratorio Analisi Chimico Cliniche, Ospedale San Donato, Arezzo, USL SUDEST \\ 2 Dipartimento Emergenza urgenza Medicina e chirurgia d'accettazione e d'urgenza, Ospedale San Donato, Arezzo, \\ USL SUDEST
}

Sepsis is a clinical syndrome, consequent to an abnormal inflammatory response to an infectious pathogenic noxa. Sepsis incidence is constantly increasing together with the average age of the population, the chronicization of many diseases, the increase in invasiveness of diagnostic procedures and treatments in the infections Related Assistance (ICA), worsened by the inappropriate use of antibiotics. Since Sepsis is a pathology with a critical evolution time-related, the earliness of the sepsis diagnosis and the promptness of the appropriate therapy are of fundamental importance to significantly improve the prognosis

The Guidance Document by Regional Technical Group to prevent the Sepsis, proposed concrete answers to the current problems relating to the subject, following three fundamental aspects: "Open the visual", "Manage Uncertainty" and "Act Promptly".

The development of tools to implement an early diagnosis in the Emergency Department, which combines clinical evaluation and laboratory tests, is a decisive aspect that influences the course of the patient with sepsis, allowing for early and targeted therapy. In this path the clinical laboratory plays an important role providing additional information to determine the direction of diagnosis and treatment.

In the proposed study we adopted the diagnostics criteria of the The Guidance Document by Regional Technical Group for patients with suspicion of Sepsis admitted at the Emergency Room of the Arezzo's Hospital. Enrolled patients have been tested to exclude organ damage and to determine together with inflammatory tests (Procalcitonin, Protein C Reactive, Blood cells count, creatinine, bilirubin, fibrinogen). In addition to the tests described, ESId (Early Sepsis Indicator), a CE marked parameter based on Monocyte Distribution Width, was determined with the UniCel DxH 900 instrument (Beckman Coulter). ESId parameter allows the measurement of the standard deviation of the volume distribution of monocytes based on morphological changes.

ESId was included in the "SEPSIS PROFILE" performed in all the patients with suspicion of sepsis.

The preliminary results are comforting, the ESId parameter, quick response test in the sepsis management process, carry information complementary to the others markers. The introduction of the ESId in the laboratory sepsis panel could offers a determinant contribute for multiparametric approach for this pathology. 


\section{REFERENCES}

1. Crouser ED, Parrillo JE, Seymour CW, Angus DC, Bicking K, Esguerra VG, Peck-Palmer OM, Magari RT, Julian MW, Kleven JM, Raj PJ, Procopio G, Careaga D, Tejidor L. Monocyte Distribution Width: A Novel Indicator of Sepsis-2 and Sepsis-3 in High-Risk Emergency Department Patients. Crit Care Med. 2019 Aug;47(8):1018-1025.

\section{PHARMACOKINETICS OF CANNABINOIDS IN CONVENTIONAL AND NON CONVENTIONAL MATRICES. LABORATORY TESTS}

\section{R. Pacifici}

National Centre on Addiction and Doping Istituto Superiore di Sanità V.Le Regina Elena 299,00161

Rome, Italy

Pharmacokinetics data on cannabinoids in different biological fluids are obtained by hyphenated techniques that identify and quantify each different drug and/or metabolite. Specifically in our laboratory we are applying ultra-high performance liquid chromatography coupled to tandem mass spectrometry due to the high sensitivity and specificity of this technique. In this concern, we have developed assays to identify and quantify cannabinoids, their metabolites and their precursors of therapeutic cannabis studied in blood, urine, oral fluid and sweat. It is possible to applied a fast and sensitive method using ultra-high performance liquid chromatography coupled with tandem mass spectrometry for analysis of $\Delta$-9-tetrahydrocannabinol, cannabidiol, their acidic precursors $\Delta$-9-tetrahydrocannabinolic acid $\mathrm{A}$ and cannabidiolic acid, cannabinol and some major metabolites of THC such as 11-nor-9-carboxy-THC, 11-hydroxy-THC, $\triangle$-9-THC-Glucuronide and THC-COOH-Glucuronide in conventional (whole blood and urine) and non-conventional (oral fluid and sweat) matrices in individual using recreational cannabis, medical cannabis or light cannabis. The advantage of using UHPLC-MS/MS is that together with THC and cannabidiol also their carboxylated acid tetrahydrocannabinol and cannabinoid acid can be detected and this is very important in case of medical cannabis. The kinetic of recreational cannabis is a very fast kinetic where cannabinoids are detected in the first minutes after smoking because recreational cannabis is only used by smoking or vaporization. THC, its glucuronide, 11-hydroxy-THC is present in blood in saliva but noting on sweat because sweat is an acidic medium and cannabinoids do not preferentially are excreted in this matrix. In case of medical cannabis since the used preparations are decoction and tea, peak of different cannabinoids is around one hour, delayed with respect to smoked cannabis. Conversely, in case of light cannabis, since this product is also smoked, the kinetics is similar to that of recreational cannabis. Another possibility to distinguish recreational cannabis from medical cannabis and light cannabis is the THC/CBD concentration ratio which is more than 10 in case of recreational cannabis, greater than 2 in case of medical cannabis and it is less than one in case of light cannabis

\section{THE CLINICAL LABORATORY IN PLASMA CELL DYSCRASIAS}

\section{G. Palladini}

Biochemistry, Biotechnology and Advanced Diagnostics Laboratory, Fundation IRCCS Policlinico San Matteo and University of Pavia, Pavia, Italy

Plasma cell dyscrasias (PCD) range from asymptomatic, pre-malignant conditions, such as monoclonal gammopathy of undetermined significance (MGUS), found in approximately $4 \%$ of the general population $>50$ years, and smoldering multiple myeloma, to overtly malignant diseases. Progression from MGUS to symptomatic conditions can be due to increase in clonal burden giving rise to overt multiple myeloma (MM) or to the ability of the monoclonal component (MC) to directly cause organ damage in monoclonal gammopathies of clinical significance (MGCS). The MC is the specific hallmark of PCDs and its identification and measurement is crucial in diagnosis, staging, assessing response to therapy and identifying relapses. 
Biomarkers of organ dysfunction and damage are used to identify symptomatic disease requiring chemotherapy. Thus, the clinical laboratory plays a key role in the diagnostic workup and management of PCDs. Most MCs are detected incidentally by serum protein electrophoresis (SPEP) during the investigation of other conditions. A MGUS phase consistently precedes MM and AL amyloidosis. Thus, when a MGUS is identified the MC should be quantified and typed and circulating free light chains (FLC) should be measured in order to assess the risk of progression. Patients with intermediate and high risk MGUS (having either nonIgG isotype, a MC concentration $>15 \mathrm{~g} / \mathrm{L}$, or abnormal FLC ratio) should be addressed to specialized hematological follow-up, aimed at timely detection of organ damage by MM (measuring hemoglobin, calcium and serum creatinine) or $\mathrm{AL}$ amyloidosis (measuring natriuretic peptides and albuminuria). When $\mathrm{MM}$ or $\mathrm{AL}$ amyloidosis are suspected a MC should be searched. The combination of SPEP and FLC quantification grants sufficient sensitivity to identify MCs in multiple myeloma, while in AL amyloidosis FLC measurement needs to be combined with immunofixation (IFE) of both serum and urine. Since MGCS are dreadful but treatable diseases usually caused by small plasma cell clones with low MC concentrations, the clinical laboratory should report all the monoclonal components identified, irrespective of their concentration. The ability of measuring FLC greatly increased our ability of monitoring PCD. Quantification of FLC identifies a MM defining event (ratio of involved / uninvolved FLC >100) and is used in staging AL amyloidosis. Assessment of response to chemotherapy in PCDs requires serum and urine IFE and FLC measurement (plus plasma cell count and evaluation of clonality in the bone marrow in $\mathrm{MM}$ and biomarkers of organ involvement in $\mathrm{AL}$ amyloidosis), and the same tests are used to identify relapse. Monoclonal antibody immunotherapy may interfere with response assessment and specific tools are needed to address this problem. More profound hematologic responses are associated with better outcomes in $\mathrm{MM}$ and $\mathrm{AL}$ amyloidosis, and new tools based on mass spectrometry identification of MCs in serum and urine and next generation sequencing or next generation flow cytometry to identify clonal disease in the bone marrow are being tested and will enter clinical practice to define minimal residual disease.

\section{INFORMATION, MISINFORMATION AND DISINFORMATION ON THE WEB}

\section{P. Pezzati}

SOD Sicurezza e Qualità, AOU Careggi Firenze

Social media, the collective of online communications channels dedicated to community-based input, interaction, content-sharing and collaboration, has the ability to combine the credibility of interpersonal persuasion with mass media, resulting in desired attitude or behavior among a large group of people. However, concerns have been raised as to the safety of social media MySpace, Facebook, Twitter, Cyworld, Pinterest and the like, since they appear to be a particularly fertile breeding ground for "fake news". While a fake news refers to news that has been entirely fabricated or made up, there are others way to mislead audiences, such as practicing "disinformation" (incorrect information) and "misinformation" (deceiving information). Both works because many people fail to recognize false information when it's presented to them. In a 2018 survey (1),organized by The UK National Literacy Trust, aimed to test the critical literacy skills, only $1.9 \%$ of children and young people overall were able to identify all news stories correctly as either real or fake (3.1\% of primary pupils, $0.6 \%$ of secondary students). This is comparable to a 2017 survey of adults (4\%). The tendency to readily accept falsehood can have serious consequences for evidence based decision making on a variety of social issues including health related questions. Patients use social media to research health information and/or health-related products, to communicate with providers and to look for peer group support. All these activities may lead to make dangerous decisions, or, on the opposite, may represent a powerful way to spread knowledge and health recommendations, according to the quality of web content. Even the way in which the content is presented and spread, can have different consequences. A recent study conducted on social media messaging on HPV vaccination(2) showed that provaccine messages report factual information, while antivaccine message appeal to emotion and may overrule logical reasons. Emotional content deliberately playing into people's basic emotions such as fear, anger or empathy ,unable to rapidly gain followers. Other social media strategies to attract followers includes: impersonation, group polarization, conspiracy, discrediting opponents and "trolling 
people online". In addition, social media have the unique capacity of amplifying news through phenomena such as echo chamber, filter bubble and moral tribes (3). All these have, as a consequence for users, a limited exposure to different views and possibly high risk of cognitive biases. Initiatives aimed to increase the quality of evidence available on web are desirable and praiseworthy, however, patients and health professional critical thinking and evidence based reasoning need to be promoted and sustained as a way to guard against the spread of misinformation in society.

\section{REFERENCE}

1. The final report. National Literacy Trust 2018 https://literacytrust.org.uk/.

2. Teoh D, 2019 ASCO EDUCATIONAL BOOK $\mathrm{p}$ 75-8.

3. Roozenbeek J., van der Linden S. Palgrave communication 2019 5:65.

\section{WHAT DO WE KNOW ABOUT PROCALCITONIN AND DOSING METHODS?}

\section{T. Sandri}

Humanitas Clinical and Research Center, IRCCS, Division of Laboratory Medicine, Rozzano, Milano

Procalcitonin (PCT) is the precursor of calcitonin, hormone synthetized within thyroid C cells. In healthy subjects, very low levels can be detected, while during bacterial infection or sepsis levels increase rapidly between 2 and $6 \mathrm{~h}$ and peak within 6-24 h. PCT levels are related to the severity of infection and can differentiate bacterial from viral etiology.

In addition to be a helpful marker for early detection of sepsis, PCT represents a useful marker for antimicrobial stewardship, leading a more appropriate utilization of antimicrobial therapy. Decreases in the marker concentration are associated with infection improvement and can lead to antibiotics discontinuation.

Different assays are available: the majority of them use BRAHMS antibodies. However, both EQAS programs and international studies have demonstrated that different methods may give slightly different results, showing harmonization problems.

Recently a new method has been introduced: the Access PCT, a chemiluminescent assay that can be run on Access and DXI platforms (Beckman Coulter). In this method antibodies different from BRAHMS are used.

We performed an evaluation of the analytical performance of the new Access PCT assay, including imprecision and sensitivity (Limit of Blank LoB, Limit of Detection LoD, Limit of Quantitation LoQ). Moreover, a comparison with the LIAISON ${ }^{\circledR}$ BRAHMS PCT ${ }^{\circledR} \|$ GEN run on the LIAISON XL analyzer was performed, using 200 fresh serum samples selected on the basis of PCT concentration: 50 samples with PCT $<0.5 \mu \mathrm{g} / \mathrm{L} ; 50$ samples with PCT $0.51-2.00 \mu \mathrm{g} / \mathrm{L} ; 50$ samples with PCT 2.01-10.00 $\mu \mathrm{g} / \mathrm{L}$ and 50 samples with PCT $>10.00 \mu \mathrm{g} / \mathrm{L}$.

Imprecision. Two patients pool (P1, P2) near the low-end clinical decision points, at 0.5 and $2.0 \mu \mathrm{g} / \mathrm{L}$ were prepared and two levels commercial quality control (BioRad Lyphocheck Specialties Immunoassay QC, L1 and L2) were measured. P1 resulted $0.58 \mu \mathrm{g} / \mathrm{L}$ with $4.46 \% \mathrm{CV}$; P2 resulted $1.87 \mu \mathrm{g} / \mathrm{L}$ with $3.6 \% \mathrm{CV}$. L1 target mean was $0.619 \mu \mathrm{g} / \mathrm{L}$ SD $0.1: 31$ determinations gave a mean value of $0.574 \mu \mathrm{g} / \mathrm{L}$, SD $0.035, \mathrm{CV} \% 6.15$. L2 target mean was $2.39 \mu \mathrm{g} / \mathrm{L}$ SD 0.28: 33 determinations gave a mean value of $2.14 \mu \mathrm{g} / \mathrm{L}, \mathrm{SD} 0.11, \mathrm{CV} \% 5.05$; no outliers were detected.

LoB, LoD abd LoQ were determined according to CLSI EP17-A2 protocol. The results showed a LoB $=0.0128$ $\mu \mathrm{g} / \mathrm{L}, \mathrm{LoD}=\mathrm{LoQ}=0.0208$, with all pool $\mathrm{CVs}$ below $20 \%$.

Methods concordance. Negative percent agreement at 0.5 e $2.0 \mu \mathrm{g} / \mathrm{L}$ was $100 \%$. Positive percent agreement was $89.3 \%$ at $0.5 \mu \mathrm{g} / \mathrm{L} \mathrm{e} 95 \%$ at $2.0 \mu \mathrm{g} / \mathrm{L}$.

These data demonstrate that the new Access PCT assay has good analytical performance. The correlation with the LIAISON PCT showed that the two methods concordantly classified all the negative subjects, while positive samples gave slightly lower results with Access PCT, result that was expected, as LIAISON is one of the methods giving higher PCT levels.

Further studies are needed to better evaluate the comparison of Access PCT with the other commercially available assays. 


\section{EXERCISE PRESCRIPTION FOR CHRONIC CONDITIONS}

\section{G. Chiaranda ${ }^{1,2}$}

${ }^{1}$ Directorate General for Person Care, Health and Welfare, Emilia-Romagna Region

${ }^{2}$ Sports Medicine and Physical Activity Promotion Unit, AUSL of Piacenza

Exercise prescription commonly refers to the specific plan of fitness-related activities that are designed in order to accomplish the needs of people affected by chronic conditions. The exercise plan relies upon exercise testing, for the objective measurement of fitness components (i.e. body composition, aerobic power, muscular strength and endurance, flexibility, coordination and balance).The customized exercise training program defines exercise Frequency, Intensity, Time, Type, total Volume and Progression (FITT-PV principle) (1). There is strong evidence that to comply with exercise prescription improves biochemical parameters, quality of life and survival in helathy people as well as in all frailty conditions and several chronic illness, including ischemic cardiomyopathy and hearth failure, diabetes, chronic obstructive pulmonary disease, osteoporosis, breast cancer. However, in order to obtain compliance, the goal of exercise prescription must be focused on patients motivation.

Due to its potential to improve health conditions and reduce healthcare costs, the exercise referral promotion is now recognized as an essential assistance level by the Italian national healthcare system (2), but its implementation is still problematic. Public health programs need exercise testing and prescription schemes sustainable and universally available. Testing must be economic, convenient for patients and easy to repeat along a several years follow up. Exercise facilities must be near to patient's home, and must include public spaces and green areas. Counseling approach must be tailored in order to sustain individual motivation. There is now the need of:

- research that focuses on economic, simple, ambulatory, long term, exercise prescription programs, that might include inexpensive and easy to perform exercise testing to evaluate and feedback patients about their fitness level. Research design should be intention to treat

- sharing of practices and policies that aim to make exercise prescription universally available

- promote knowledge and attitudes towards exercise prescription among healthcare providers

The Emilia-Romagna regional council act D.G.R. n. 2127/2016, and subsequent operational protocols, design a framework for exercise prescription and referral that involves public healthcare system and recognized private gyms with a kinesiologist with a specialist graduate degree in preventive and adapted motor activity. Outpatients with neurological or osteoarticular disorders are referred to group exercise sessions. Those with cardio respiratory or metabolic conditions are evaluated in sports services and then are prescribed with tailored supervised gym or autonomous open air exercise programs. An easy to perform, perception based submaximal walking test has been developed. Effectiveness of this scheme is supported by observational studies (3) and preliminary data from RCTS (4) performed by regional universityiesand health institutions.

\section{REFERENCE}

1. ACSM'S Guidelines on exercise testing and prescription, $9^{\text {th }}$ edition.

2. DPCM 12 gennaio 2017, allegato 1, Gazzetta Ufficiale della Repubblica Italiana - Supp. n.15.

3. Heart. 2016;102:1902-1908.

4. BMC Cardiovasc Disord 2018;18:98.

\section{BENEFITS AND RISKS OF STANDARDIZATION, HARMONIZATION AND CONFORMITY TO OPINION IN CLINICAL LABORATORIES}

\section{Naugler}

Professor, University of Calgary

The trend toward increasingly larger laboratory networks has resulted in the desire to standardize platforms and procedures among laboratories. This trend is also driven by administrators seeking to achieve resource efficiencies, and to meet the changes brought about by computerization and the adoption of electronic medical records (1). Although conformity and standardization of laboratory networks may improve testing efficiencies 
and patient care outcomes, this approach can also have serious drawbacks. In most instances standards do not exist and so 'standardization' becomes conformity to an arbitrary opinion and can predispose to system failure, reduced competitiveness, constraint of innovation and diminished patient care quality and outcomes (1-3). The application of standardization in clinical laboratories has also unmasked the distinct philosophical approaches of laboratory physicians and scientists (i.e., "professionalism") towards standardization initiatives compared to laboratory administration and managers (i.e., "managerialism"). Managerialism argues that administrative oversight of laboratories is needed to ensure proper 'control' of the system, which cannot be entrusted to individuals. The social purpose of managerialism is therefore not primarily patient-centered. In contrast, professionalism is a patient-centered approach whose social purpose is primarily focussed on patient outcomes. Professionalism is more closely aligned with right-brain thinking, creativity, emotional intelligence, critical thinking and external motivators, all attributes required for innovation $(4,5)$.

\title{
REFERENCE
}

1. Church DL, Naugler C. Benefits and risks of standardization, harmonization and conformity to opinion in clinical laboratories. Crit Rev Clin Lab Sci 2019 Aug;56(5):287-306.

2. Church J, Gerlock A, Smith DL. 2018. Neoliberalism and Accountability Failure in the Delivery of Services Affecting the Health of the Public. International Journal of Health Services 48:641-662.

3. Mannion R, Braithwaite J. 2012. Unintended consequences of performance measurement in healthcare: 20 salutary lessons from the English National Health Service. Internal Medicine Journal 42:569-574.

4. Judson TJ, Volpp KG, Detsky AS. 2015. Harnessing the Right Combination of Extrinsic and Intrinsic Motivation to Change Physician Behavior. JAMA 314:2233-4.

5. Phipps-Taylor M, Shortell SM. 2016. More Than Money: Motivating Physician Behavior Change in Accountable Care Organizations. Milbank Q 94:832-861.

\section{DETECTION AND EVALUATION OF AUTOIMMUNE DISEASES AUTO-ANTIBODIES ON A NEW AUTOMATED CHEMILUMINESCENT SYSTEM}

\author{
M. Nuccetelli, F. Gisone, F. Romani, S. Bernardini \\ Department of Experimental Medicine and Surgery, Tor Vergata University Hospital, Rome, Italy
}

Autoimmune diseases are disorders in which the immune system fails to distinguish self form non-self antigens. They are characterized by the presence of specific autoantibodies; among them antinuclear antibodies (ANA) are the most representative and their detection is of great importance in laboratory diagnosis of systemic immune diseases for screening and monitoring of Systemic Lupus Erythematosus (SLE), Progressive Systemic Sclerosis (PSS), Mixed Connective Tissue Disease (MCTD), Sjögren Syndrome (SS), Polymyositis (PM) and Primary Biliary Cirrhosis (PBC). In general, ANA can be divided into extractable nuclear antigens (ENA), non-extractable nuclear antigens and cytoplasmatic antigens; their presence is evaluated by enzymatic immunoassays (Enzyme Linked Immunosorbent Assay, ELISA or Chemiluminescence Assay, CLIA) and by indirect immunofluorescence (IIF) using HEp-2 cells, a human epithelial cell line, as an antigen source. Indirect immunofluorescence represents the reference test. Our study was focused on the comparison of ANA detection between consolidated diagnostic kits and a new commercial automated chemiluminescent assay provided by Snibe Diagnostic. The quantitative determination of IgG antibodies was performed on a total of 131 sera for the following tests: ANA screen, ENA screen, SSA, SSB Scl-70, CENP, Sm, Sm/rnp, Jo1, dsDNA, M2-3E. For each sample we had the immunofluorescent corresponding pattern to use as control for the positivity or negativity of the autoantigens detected. Our results showed the following discordances compared to our methodologies: two negative CENP samples (Snibe Clia Assay lacks CENP B antigen); two positive Scl 70 samples (we are evaluating the antigen source), two negative SSA samples (Snibe Clia Assay lacks SSA 52kDa subunit), two negative M2-3E samples (a possible explanation is the disaggregation on the magnetic beads, of the complex formed by pyruvate dehydrogenase, 2-oxo glutarate dehydrogenase and 2oxo-acid dehydrogenase), six dsDNA negative samples not confirmed by indirect immunofluorescence on Crithidia Luciliae. In conclusion, $10.6 \%$ of samples analyzed with Snibe kits showed different results. Most of them are related to the lack of the antigen in the detection assay (SSA 52kDa and CENP A) and the others 
maybe are related to the source of the antigens (most of them are native and could be less stable or with a lower recovery in respect to the recombinant antigens). Our suggestion for improving the performance of the tests is first of all adding the lacking antigens in the assay; then taking into account the possibility of changing the antigen source (native are less stable and pure, so can introduce cross reactions with not specific antigens). Finally, we suggest also the introduction of $\mathrm{Pm} / \mathrm{Scl}$ autoantibodies assay, useful for the overlap syndrome diagnosis.

\title{
EVALUATION OF THE HEPATITIS B TEST PANEL ON MINDRAY CL 1200i SYSTEM AND COMPARISON OF DIAGNOSTIC RESULTS
}

\author{
M. Pieri, S. Grelli, S. Bernardini \\ Department of Experimental Medicine and Surgery, University of Rome 'Tor Vergata', Rome, Italy
}

Background: Hepatitis B virus (HBV) infection is a public health problem worldwide. People with chronic hepatitis B infection have an increased risk of developing cirrhosis or hepatocellular carcinoma (1). The lack of global HBV vaccination coverage, the absence of sufficient protection in part of the vaccinees and the failure of protection in 10-30\% of newborns from highly viremic mothers results in ongoing global transmission of hepatitis B (2). In worldwide, two billion people have been infected with HBV, and more than 350 million of infected patients are chronic carriers of HBV. The various guidelines for hepatitis $\mathrm{B}$ vaccination, booster policy and post liver-transplant management imply that the measurement of anti-HBs levels by different assays is accurate and consistent, yielding comparable quantitative results in a range of laboratories. During these years many automated immunoassay analyzers with high sensitivity, specificity, and throughput have been developed.

The aim of this study was to evaluate the performance of Mindray CL 1200i for HBsAg, Anti-HBs, HBeAg, Anti$\mathrm{Hbe}$, Anti-HBc in comparison with ABBOT Alinity System from patients of the "Tor Vergata" University Hospital. Methods: patients with chronic hepatitis B and control patients from "Tor Vergata" University Hospital were assessed using Mindray CL 1200i and ABBOT Alinity System. We evaluate precision, linearity, carry over, throughput of Mindray CL 1200i and compare the obtained data with Abbot Alinity system. Precision was assessed using $3 \times 5$ ( 3 replicates for 5 days) over a relatively long time interval (at least 2 weeks) for normal and high CQI. The linearity was determined by using serial dilutions of a high sample plasma with a low concentration sample plasma samples in order to obtain parameter levels in the range from $0 \%$ to approximately $100 \%$ for HBsAg and Anti-HBs.

Results: we verified the repeatability and precision of the test method using a quick protocol to check manufacture's product declaration. The variation coefficient (CV) obtained for all the analytes tested between run and within run show a good acceptability compared with those declared by the manufacturer. The linearity studies showed excellent performance for HBsAg and Anti-HBs (R2 0.993 and R2 0.992; respectively). The carryover was detected in triplicate alternate measurements of a sample with a high and low test result, the data obtained showed no carryover for HBsAg.

Conclusions: the precision data are good and confirm the CVs declared by the manufacturer and the comparison with the Abbott system shows a significant correlation. The Mindray CL 1200i analyzer is suitable for the determination of HBsAg, Anti-HBs, HBeAg, Anti-Hbe, Anti-HBc in routine analysis.

\section{REFERENCE}

1. Liao, C.C., C.W. Hsu, P.W. Gu, C.T. Yeh, S.M. Lin, et al. (2015) Comparison of the elecsys HBsAg II assay and the architect assay for quantification of hepatitis B surface antigen in chronic hepatitis B patients. Biomed $\mathrm{J}$ 38(3):250-6.

2. Raven, S., J. Hautvast, J.V. Steenbergen, R. Akkermans, C. Weykamp, et al. (2017) Diagnostic performance of serological assays for anti-HBs testing: Results from a quality assessment program. J Clin Virol 87:17-22. 


\title{
METHODS AND PRACTICAL ASPECTS OF THERAPEUTIC MONITORING: MANAGEMENT OF DRUG INTERACTIONS
}

\section{F. Dal Piaz}

Dipartimento di Medicina, Chirurgia e Odontoiatria "Scuola Medica Salernitana", Università degli Studi di Salerno

One of the main pitfalls in the choice of the dosage of a drug to be administered to a patient is the simultaneous intake of other substances, that may somehow modify the absorption, metabolism or efficacy of the drug. This problem, well known to pharmacologists, is often analyzed during clinical trials, especially for those drugs often used in poly-therapies (for example in the case of many antiepileptic drugs or for some antiblastic chemotherapics (1)); moreover, where the metabolism of a drug is well characterized and its excretion mechanism is known, it is possible to predict with discrete precision the effect of the simultaneous intake of substances with a similar metabolic fate. Nonetheless, there is a wide range of interferences between drugs, or between a drug and other substances, which escape a priori evaluations or that are subject to a significant interindividual variability. A paradigmatic example is what is often observed in subjects who use food supplements of herbal origin: many compounds present in these products - which are often not well characterized from the point of view of chemical composition - can significantly modify the absorption and bioavailability of orally administrated drugs (2).

All this can result in a lack of or reduced therapeutic efficacy or, on the other hand, in overdoses and unexpected toxicity; the effects of such alterations can also be serious, especially in fragile subjects (primarily elderly and infants), wich are often subjected to multiple therapies and preventive treatments.

The availability of methods and procedures designed to predictively assess the risk of interactions - or at least to allow verifying their effects early - on individual subjects is therefore a key requirement to increase the likelihood of success of several therapeutic approaches. The combined and complementary use of pharmacogenomic analysis and therapeutic monitoring, coupled with an in-depth understanding of the pharmacology of different substances, is emerging as the most effective strategy to design the optimal therapeutic protocol to be applied to each patient, in order to minimize the consequences of interference between different substances (3). However, there is a still not completely satisfied request for innovative approaches capable of providing more precise information, and of ever deeper knowledge about the mechanism of action and the metabolism of multiple bioactive substances.

\section{REFERENCE}

1. Lauren A. et al. (2018) Prevalence of drug-drug interactions in oncology patients enrolled on National Clinical Trials Network oncology clinical trials. BMC Cancer 18: 1155.

2. Sprouse AA \& van Breemen RB. (2016) Pharmacokinetic Interactions between Drugs and Botanical Dietary Supplements. Drug Metab Dispos 44: 162-71.

3. de Leon J. (2019) Personalizing dosing of risperidone, paliperidone and clozapine using therapeutic drug monitoring and pharmacogenetics. Neuropharmacology S0028-3908: 30197-202.

\section{DEMOGRAPHIC FRAGILITY AND INFERTILITY: THE CONTRIBUTION OF LABORATORY MEDICINE}

\author{
V. D'Argenio ${ }^{1,2,3}$ F. Cariati ${ }^{1,3}$, R. Tomaiuolo ${ }^{2,3}$ \\ ${ }^{1}$ CEINGE-Biotecnologie Avanzate scarl, Via Gaetano Salvatore 486, 80145 Napoli, Italy \\ ${ }^{2}$ Dipartimento di Medicina Molecolare e Biotecnologie Mediche, Università degli Studi di Napoli Federico II, Via Sergio \\ Pansini 5, 80131 Napoli, Italy \\ ${ }^{3}$ KronosDNA srl, spinoff of Università Federico II, Napoli, Italy
}

Today, infertility is considered a major public health issue: it has been estimated that approximately 1 out of 6 people worldwide suffers from infertility during the reproductive lifespans. This number is expected to grow up in a near future due to the increased age of both partners at first pregnancy and also to unhealthy lifestyle habits. Thus, the management of health reproduction will be ever more important for healthcare providers. 
As with all areas of health - to stay healthy people needs to be empowered with knowledge - the provision of accurate fertility information must become mandatory to increase fertility awareness. Women and men are unaware of their own fertility potential, the constraints on their fertility, the signs, symptoms or preventable causes of fertility problems (1). In particular, there is a lack of awareness of individual variation in terms of how fertility declines with age; globally, people are delaying the age of first conception for several reasons (2), but female and male fertility declines with age based on a combination of genetic and lifestyle factors $(3,4)$. Understanding how individual fertility may decline with age and the modifiable lifestyle factors, such as diet and alcohol consumption, is a huge opportunity for increasing fertility awareness and ensuring people are able to make well informed reproductive choices.

In this context, it is important to underline that the reproductive systems of both partners have to work properly and in a precise combination to get a pregnancy; thus, infertility assessment requires the simultaneous evaluation of the two members of a couple to be effective.

Considering all the above, laboratory medicine is expected to have a key role in reproductive-related issues. Indeed, thanks to the availability of sensitive and highly performing technics for the detection of DNA alterations in several genes simultaneously and starting from low amount of DNA, molecular analyses are becoming most widely used, also in reproductive medicine. First of all, a molecular evaluation of both partners can be useful to identify the causes of male and/or female infertility, thus providing useful information for the clinical management of the couple. In addition, it is possible to identify genetic diseases transmissible to the offspring and, consequently plan antenatal testing in order to avoid the birth of affected child. Finally, molecular testing can support the decision for the most proper assisted reproductive technique. Thus, the integration of molecular analyses at different steps of the infertile couples journey, based on couples personal and familial history, is able to improve their reproductive outcomes. Nevertheless, to date the use of molecular analyses in this field is still fragmented and cumbersome. However, stratifying the population, through the identification of risk factors and diseases that may be present, allows the planning of targeted diagnostic-therapeutic programs (5).

\section{REFERENCE}

1. Daniluk JC, Koert E. The other side of the fertility coin: a comparison of childless men's and women's knowledge of fertility and assisted reproductive technology. Fertil Steril 2013;99:839-46.

2. Mills $M$, Rindfuss RR, McDonald $P$, et al. Why do people postpone parenthood? Reasons and social policy incentives. Human Reproduction Update 2011;17:848-60.

3. Everywoman, J. Cassandra's prophecy: why we need to tell the women of the future about age-related fertility decline and 'delayed' childbearing. Reprod BioMed Online 2013;27:4-10.

4. Cariati F, D'Uonno N, Borrillo F, et al. Bisphenol a: an emerging threat to male fertility. Reprod Biol Endocrinol 2019;17:6.

5. Cariati F, D'Argenio V, Tomaiuolo R. The evolving role of genetic tests in reproductive medicine Journal of Translational Medicine, under 2th review.

\section{ARE THERAPEUTIC REFERENCE RANGES ALSO VALID FOR FRAGILE POPULATIONS? THE ELDERLY POPULATION}

\section{U. de Grazia}

Senior biologist, BiolSci, PhD, Clinical Pathologist, SSD Laboratory of Neurological Biochemistry and Neuropharmacology, Fondazione I.R.C.C.S. Istituto Neurologico Carlo Besta, Milano, Italy

Elderly people are particularly susceptible to adverse drug reactions because of multimorbidity, the high number of medications used by this population and age associated changes in pharmacokinetic and pharmacodynamic properties. The drug doses that are usually prescribed for younger adults may be too high for old individuals. In addition, it is important to recognize the heterogeneity of drug response in the elderly. Therefore, there are no simple rules for prescribing that may apply to the entire elderly population. Rather the dose has to be determined individually considering particularly the reduction in body weight and renal elimination in a given patient. Pharmacokinetic guidelines may be used to calculate doses that result in drug 
serum levels in the aged equivalent to younger adults. But this procedure neglects changes in sensitivity to drugs. Hence, starting from a smaller initial dose than used in younger adults the dose should be titrated to a clearly defined therapeutic response. It would be convenient to use therapeutic drug monitoring to adjust dosage in old-age patients. The brain is an especially sensitive drug target in old age. Psychotropic drugs, anticonvulsants, and centrally acting antihypertensive may impede intellectual function and motor coordination. Hence drugs should be used restrictively in geriatric patients. A growing number of scientific paper has been published on this topic. All studies converge on the idea that drug therapy must be "personalized" in older people According to literature this goal may be achieved changing drugs dosing, avoiding multidrug exposures, choosing the right drug for the right person, introducing a more widespread use of pharmacogenomics to explain and eventually predict pharmacokinetics and pharmacodynamics changes. This implies that there are not established therapeutic reference ranges for drugs in the elderly population but instead the approach to a more safe and effective drug therapy is carried out by dose changing, although is already known that most of drugs concentrations are influenced by age. Ultimately, the result of this strategy is to try to bring the patient's blood drug levels back within the therapeutic ranges of the adult with less than 65 years by the mean of therapeutic drug monitoring. In this way it becomes problematic to establish specific values for the elderly population. Studies are needed on a large number of patients to understand whether it is possible or not to validate specific intervals for elderly people. Furthermore, many studies today are no longer focused on a single age group (> 65 years) but this group is further subdivided into other subgroups (e.g. $>75$ and $>81$ ) since the accumulation of new data is always making this difference is more evident. However, robust drug serum concentration-effect relations for the elderly are lacking and therapeutic target concentrations are not well established for this age group. As a matter of fact, it may be difficult if not impossible to define such target concentrations for the elderly because of the marked inter individual variation in this segment of the population.

\section{BIBLIOGRAFIA}

- $\quad$ Miller SW. Therapeutic Drug Monitoring in the geriatric patient. Clinical pharmacokinetics, 6th edition. JE Murphy, editor. 2017, American Society of Health-System Pharmacists.

- Scibona P et al. Individualization of drug therapy in older people. Rev in Clin Gerontol 2014;24:145-157.

- $\quad$ Turnheim K. Drug therapy in the elderly. Exp Gerontol 2004;39:1731-1738.

\section{USEFULLNESS OF THERAPEUTIC DRUG MONITORING IN CLINICAL PRACTICE}

\section{A. Di Paolo}

Dipartimento di Medicina Clinica e Sperimentale, Università di Pisa, UO Farmacologia Clinica, Azienda Ospedaliero Universitaria Pisa

The ultimate goal of modern medicine is to improve the survival and the quality of life of patients who may benefit from the therapeutic effect of the treatments without experiencing adverse drug reactions (ADR). Although the efforts and the knowledge gathered during drug development and subsequently in daily clinical routine, several factors may influence the efficacy and the tolerability of pharmacological therapies. Some of these factors are linked to distinctive characteristics of patients, such as age, gender, organ and system functions, allelic variants of genes coding for enzymes (i.e., liver CYP isoforms) and transmembrane transporters (i.e., members of ABC and SLC families), to the disease and co-morbidities, and finally to the presence of other drugs (with the potential risk of drug-drug interactions) or therapeutic interventions (as well as renal replacement therapies). Whatever the involved factor/s could be, known or presumed, the expected positive outcome of the treatment can be disregarded because of the onset of therapeutic failures or toxicities. These conditions represent the rational prerequisite for the development and application of therapeutic drug monitoring (TDM), which aims to identify the relationship between the dose administered and the effective concentrations in biological matrices for every patient. Thanks to the application of TDM protocols in clinical practice, it is possible to personalize the dosage of drugs in a relatively short time compared to that necessary for a therapeutic failure or toxicity to occur, hence maximizing the therapeutic benefit experienced by patients and protecting them from ADR. Indeed, several studies have demonstrated that TDM protocols can increase 
the success rate of therapies, even in fragile population, while reducing the ADRs and the need for hospitalization. However, the correct application of TDM requires the careful evaluation of numerous factors, as well as the clinical conditions of the patients, the severity of the disease, the pharmacological characteristics of the drug (i.e., therapeutic index and range), and, as listed above, the presence of comorbidities and concomitant therapies. Under this spectrum of possible critical factors, the laboratory would offer reliable and reproducible tests, with a turn-around-time sufficiently rapid to be used even in emergencies (when required), and with a low cost. Since the last years, the number of drugs that can undergo TDM is still increasing, and this is mainly due to the development of kits suitable for chromatographic instruments, whose diffusion is growing across laboratories. However, a further step will be the systematic application of validated algorithms to predict individual doses based on TDM findings, a procedure that today is available for a few drugs. This approach will definitively represent the final answer for clinicians because it will enable the personalization of pharmacological therapies. In conclusion, several disciplines and professionals may contribute to the elaboration of TDM protocols that represent a valid and useful tool for personalized medicine.

\title{
THE DRONES IN THE NEW VISION OF HEALTH CARE AND IN THE EVOLUTION OF LABORATORY MEDICINE. FIRST EXPERIENCE IN ITALY
}

\author{
M. D'Amora', T. Trenti ${ }^{2}$, L. Atripaldi3, M. Mussap4, G. Canonico'1, A. Contina', M.E. Gragnaniello', U. \\ Atripaldi ${ }^{3}$
}

${ }^{1}$ UOC Medicina di Laboratorio PP.OO: "San Paolo-Loreto Crispi" AsI Napoli 1 Centro

${ }^{2}$ Dipartimento Medicina di Laboratorio AOU Modena

${ }^{3}$ UOC Biochimica Clinica AORN dei Colli Napoli

${ }^{4}$ Medicina di Laboratorio Dipartimento di Scienze Chirurgiche Università di Cagliari

Medicine at this particular current moment is experiencing a major transformation due to the exponential development of digital and emerging new technologies. Ultramodern technological devices are present in most Italian and worldwide hospitals, now even drones can contribute to the further evolution of healthcare, both hospital and territorial. The drones today, thanks to the evolution of information technology applied to innovative technologies, can be used in the connection between hospitals and other health facilities for the transport, even in urgency, of defibrillators, drugs, blood bags and / or blood products and other biological material (test tubes of blood, urine, tissue samples, etc.) even over long distances (max. $100 \mathrm{~km}$ ). The drone, equipped with a safety container that meets all national and international standards for the transport of biological substances, can be loaded manually or through a fully automated station that allows dedicated and specially trained operators to load the container by inserting it directly in the appropriate adapter of the drone itself without any manual contact with the APR, to then make it take off also through a specific application (App) on a smartphone. The Study Group on Management and Organizational Innovation in Laboratory Medicine proposed to $\mathrm{SiBioC}$ an experimental project on process innovation in Laboratory Medicine and Health Services entitled "infrastructure and mobility with Drones in a digital ecosystem" that has achieved the patronage of Company on 25 October 2018. The project was called "Philotea" and the SIBioC Delegate of the Campania Region was identified for the coordination of all related activities. The experience that took place for the first time in Italy on 7 March 2019, with the authorization of the National Civil Aviation Authority (ENAC) and of the other authorities in charge, established the connection of two hospital hubs of a Company Health of the metropolitan city of Naples (also known for the great difficulties of connections linked to constant vehicular traffic) by testing thirty parameters (hematology, coagulation and clinical chemistry tests) before and after the flight to verify the perfect correspondence to the indispensable precision and accuracy laboratory tests to guarantee first and foremost patients and subsequently also health professionals. The evaluations carried out allow us to positively evaluate this first experience, followed by others, even in different weather conditions. 